\title{
Temporal evolution of a patient with a spinal dural arteriovenous fistula on serial MRI
}

\author{
Michael G. Kim ${ }^{1}$ - Seung W. Jeong ${ }^{2} \cdot$ Elena Solli ${ }^{3} \cdot$ Anubhav G. Amin ${ }^{1} \cdot$ Jennifer S. Ronecker ${ }^{1} \cdot$ Shalabh Bobra $^{4}$
}

Received: 11 November 2017 / Revised: 17 December 2017 / Accepted: 19 December 2017

(c) International Spinal Cord Society 2018

\begin{abstract}
Introduction A spinal dural arteriovenous fistula is a rare type of vascular malformation. If left untreated, these fistulas can result in significant neurological deficits secondary to spinal cord infarct or hemorrhage.

Case presentation A 70-year-old female with a longstanding history of episodic progressive bilateral lower extremity weakness and sensory disturbances was previously misdiagnosed with multiple sclerosis. Imaging revealed a T2 signal change from $\mathrm{T} 7$ to the conus with associated signal change and she subsequently underwent a T10-L1 laminectomy for clip ligation of a spinal dural arteriovenous fistula. Here we present the clinical and radiographic progression of one patient with a spinal dural arteriovenous fistula.

Discussion Spinal dural arteriovenous fistulas are a rare but treatable cause of myelopathy, so it is important to understand its natural progression and radiologic findings as it is frequently misdiagnosed.
\end{abstract}

\section{Introduction}

Spinal vascular malformations (SVMs) are a heterogeneous group of blood vessel disorders that account for $3-4 \%$ of all space-occupying lesions affecting the spinal cord and 1-2\% of all neurologic vascular pathologies [1-3]. SVMs are classified into four types based on location, affected population, and radiographic appearance [4]. Spinal dural arteriovenous fistulas (SDAVFs), also known as type $1 \mathrm{SVMs}$, are the most common form of SVMs, accounting for approximately $70-80 \%$ of all spinal vascular lesions $[2,3$, 5-8]. However, SDAVFs are relatively rare with an estimated incidence of 5-10 per million per year and are frequently misdiagnosed as tumor, myelitis, polyneuropathy, and polyradiculopathy, with patients often waiting 1-2

Michael G. Kim

Michael.Kim@wmchealth.org

1 Department of Neurosurgery, Westchester Medical Center, Valhalla, NY, USA

2 New York Medical College, Valhalla, NY, USA

3 Department of Neurosurgery, Rutgers New Jersey Medical School, Newark, NJ, USA

4 Department of Radiology, Westchester Medical Center, Valhalla, NY, USA years before a correct diagnosis is identified [9-16]. This is due to the non-specific clinical features of SDAVF as well as the variable imaging findings associated with it. Here, we present serial imaging from a case of SDAVF, in which we were able to clinically and radiographically observe the development of SDAVF symptoms and imaging findings, with subsequent progression to congestive myelopathy and infarction over a period of approximately 8 years.

\section{Case}

A 70-year-old female with a diagnosis of multiple sclerosis initially presented as a transfer from an outside hospital with magnetic resonance imaging (MRI) findings of T2 signal change within the spinal cord from $\mathrm{T} 7$ to the conus with associated serpiginous vessels concerning for a SDAVF (Fig. 1). She had a 2 year history of stepwise progressive bilateral lower extremity weakness, numbness, parasthesias, and dysesthesias. She had multiple episodes attributed to multiple sclerosis throughout these 2 years in which she would develop acute weakness, causing her to fall. She would improve after each episode with a short course of steroids and would then be subsequently discharged to a rehabilitation center. However, her strength did not return to her previous baseline after each of these episodes. She had 


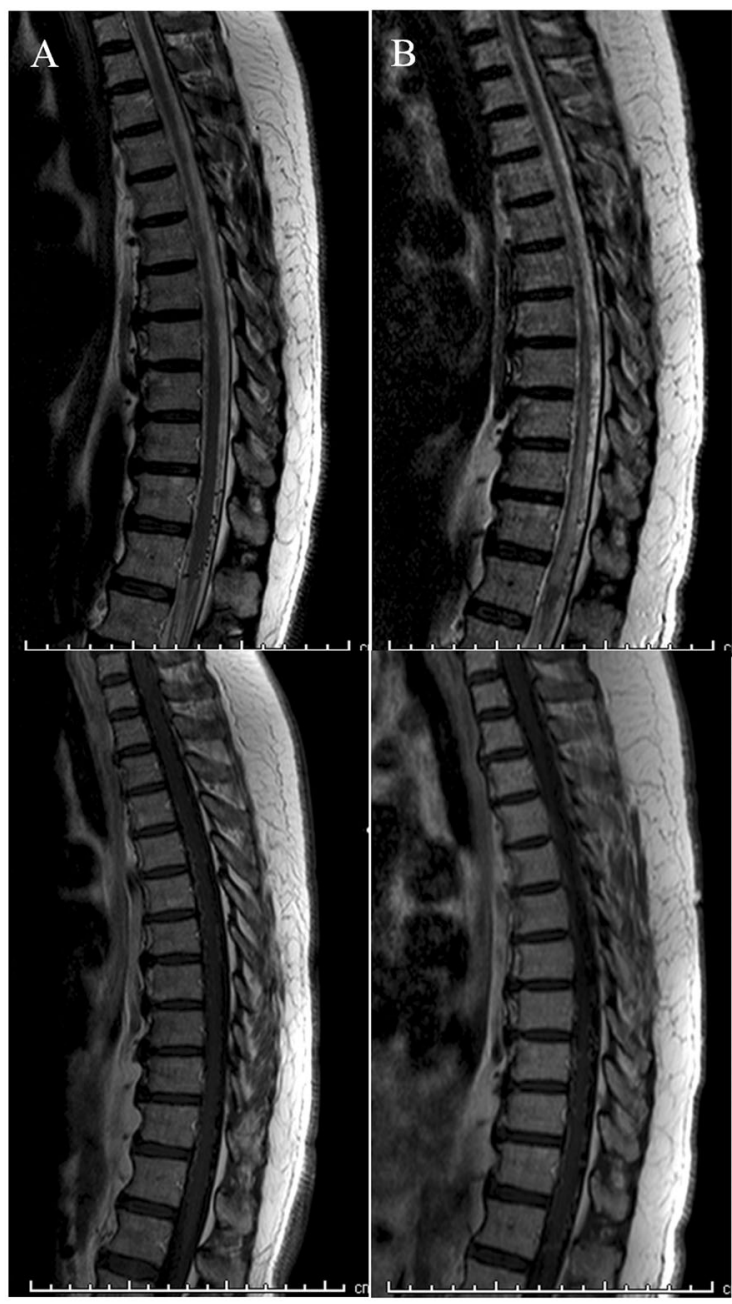

Fig. 1 The T2 weighted and T1 post-contrast sequences from an MRI obtained 8 years prior (a) shows dilated $\mathrm{T} 2$ flow voids along the dorsal aspect of the spinal cord near the conus with mild contrast enhancement of these vessels. There is no evidence of spinal cord edema or enhancement. The imaging obtained 3 years prior (b) shows edema extending from the conus to approximately $\mathrm{T} 9$ with no evidence of

previously been able to walk without assistance, but then required a cane and eventually a walker.

The patient presented to us after having another one of these episodes. Her exam at that time was an L1 ASIA D with bilateral lower extremity weakness with hip flexion $3 / 5$ and the other muscle groups a $4 / 5$. She had signs of myelopathy with sustained clonus in her left lower extremity. She had decreased rectal tone and a sensory level at L1 with decreased sensation to light touch. Given her progressive decline and MRI findings consistent for a SDAVF, the patient underwent a spinal angiogram. However, this exam was limited due to technical difficulties given her anatomy and atherosclerotic disease, and it was therefore aborted in favor of obtaining further noninvasive imaging. A CT angiogram of the thoracic and lumbar spine showed a prominent right-sided vessel arising from the T12-L1 level

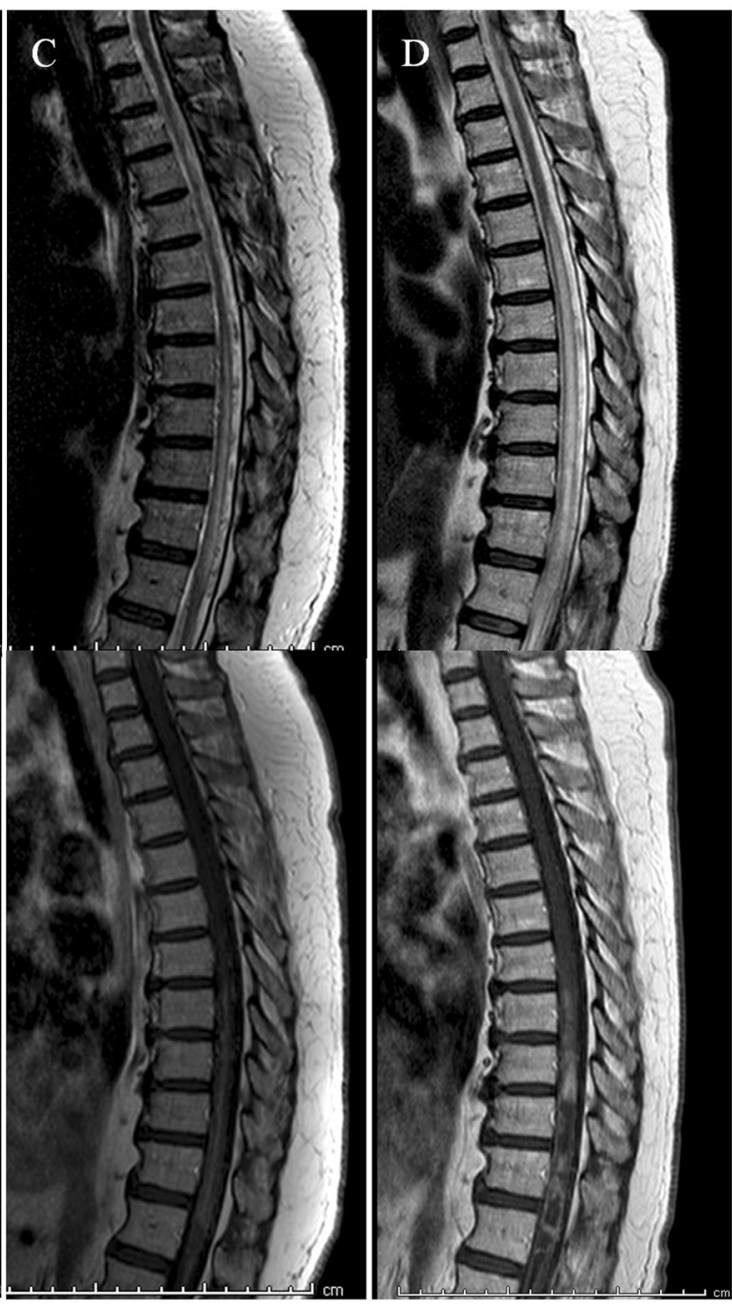

spinal cord enhancement. The imaging obtained 6 weeks prior (c) shows worsening spinal cord edema extending to $\mathrm{T} 7$ with patchy enhancement of the spinal cord. The final imaging (d) shows worsening edema extending to T5 with expansion of the lower thoracic spinal cord and avid enhancement from $\mathrm{T} 7$ to the conus, concerning for spinal cord infarction

coursing superiorly towards a tangle of vessels seen at the T11-T12 level. At this time, we deemed her prior diagnosis of multiple sclerosis as incorrect given the radiographic evidence of a SDAVF.

Surgery was offered to the patient, but she refused any further imaging or treatment and was discharged to an acute rehabilitation facility. She then returned approximately 1 month later for her scheduled follow up. She had become acutely weak 1 week after she had left the hospital and her exam had declined to a T10 ASIA B with bilateral lower extremity paraplegia and a T10 sensory level below which she could only feel dull deep pressure. Her deep tendon reflexes were absent in her lower extremities and she now had bladder and bowel incontinence. An MRI revealed an interval increase in the extent of the abnormal T2/STIR signal with patchy avid enhancement on $\mathrm{T} 1$ which now 


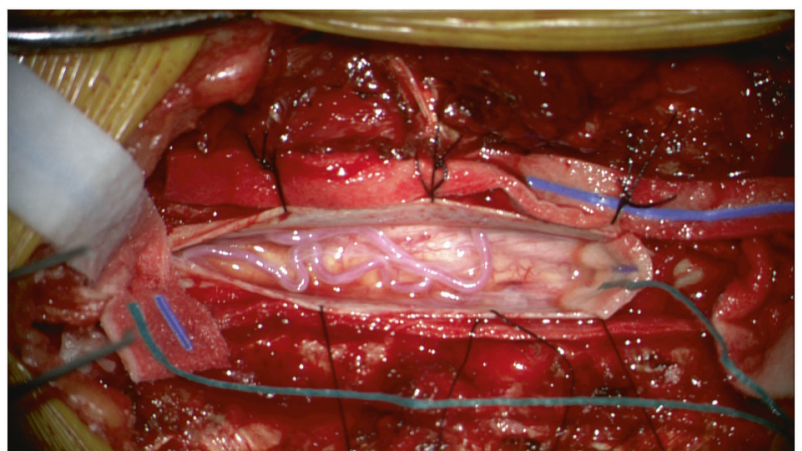

Fig. 2 An intraoperative photograph showing dilated venous structures on the dorsal aspect of the spinal cord after a T10-L1 laminectomy had been performed

extended from $\mathrm{T} 5$ to the conus with expansion of the spinal cord. There was high signal intensity in the diffusionweighted sequences from T10 to the conus concerning for cord infarction.

The patient then underwent a T10-L1 laminectomy and clipping of SDAVF. Upon initial inspection, the spinal cord near the conus appeared abnormal, consistent with recent infarct and necrotic changes. A large engorged arterialized vein was visualized on the dorsal surface of the spinal cord (Fig. 2). It was followed inferiorly and was found to be connected to a right radicular meningeal artery at the T12L1 level. Indocyanine green was injected to confirm the site of the fistula. Two clips were then applied to disconnect the fistula site and a second indocyanine green injection revealed no arterial supply to the dorsal fistula. The patient had an uneventful postoperative course and was then discharged to an inpatient acute rehabilitation center on postoperative day 8. Unfortunately the patient's neurologic status did not improve in the immediate postoperative period.

\section{Discussion}

\section{Clinical features and pathophysiology of spinal dural arteriovenous fistulas}

The pathophysiology of SDAVF is defined by venous hypertension caused by the abnormal connection between the radicular artery and radicular vein within the dura [11, 17-22]. The arterialization of the perimedullary venous plexus leads to a subsequent loss of the arteriovenous gradient, resulting in decreased venous drainage, venous congestion, edema, hypoperfusion, and ultimately ischemia [3, $17,18,23,24]$. The etiology of SDAVF is generally idiopathic in most cases, but acquired cases following surgery or trauma have been reported [17, 25]. They occur most commonly in men over the age of 40 with a male to female ratio of $4: 1$, and are predominantly found in the thoracolumbar region $[17,20,26]$.

SDAVF presents with relatively non-specific symptoms and subtle radiographic findings, making it difficult to diagnose, with median time to diagnosis ranging between 12-44 months from symptom onset [10-12]. One study found that $43 \%$ of patients were severely disabled by the time the SDAVF was found [12]. Symptoms may include weakness, gait disturbances, numbness, parasthesias, pain, sphincter dysfunction, erectile dysfunction, and urinary retention [11-13, 27]. The most common initial presenting symptom of SDAVF is progressive motor weakness, present in $85-95 \%$ of patients $[3,8,11,17,18,20,26]$. These neurologic symptoms are progressive with time and often ascend from the lower extremities due to the fact that the conus is involved in the vast majority of patients as a result of orthostasis $[11,12]$. It has been estimated that if left untreated, $50 \%$ of patients will become severely disabled within 3 years of symptom onset and less than $10 \%$ will be able to walk independently after 3 years $[17,28]$. However, $5-15 \%$ of patients with SDAVFs develop acute thrombosis of the pathologic veins that drain the fistula, resulting in acute exacerbation of symptoms and rapidly progressive myelopathy $[17,29,30]$.

SDAVF can be treated either endovascularly or surgically. Microsurgical obliteration of the fistula has proven to be highly effective with a reported success rate of approximately $98 \%$ and improvement in motor function seen in $82.2 \%$ of patients [8, 20, 25, 26, 31, 32]. Endovascular treatment has yielded slightly less successful results, with $69 \%$ achieving complete obliteration of the fistula, 25\% requiring additional procedures, and $15-20 \%$ experiencing recurrence $[27,33]$.

\section{Imaging modalities and features of spinal dural arteriovenous fistulas}

Essential diagnostic imaging modalities for SDAVF include MRI, magnetic resonance angiogram (MRA), and digital subtraction angiogram (DSA). When subtle features suggestive of SDAVF are found on MRI, MRA may then be used to better visualize and localize the fistula. DSA is subsequently used to confirm the diagnosis.

On T2-weighted MRI, key imaging findings include centromedullary hyperintensity with peripheral hypointensity and flow voids, respectively representing cord edema and dilated perimedullary veins [3, 15, 24, 34-37]. Occasionally, these veins become difficult to visualize due to compression by cord swelling [24]. The central T2 hyperintensity frequently involves the conus due to orthostasis and one study found that $81 \%$ of patients displayed involvement $[24,38]$. If a patient's initial non-contrast MRI does not clearly show a SDAVF when clinical suspicion remains 
high, gadolinium enhanced MRI may aid in visualization. With contrast, parenchymal enhancement is frequently observed, likely representing infarction or ischemia from the venous hypertension [36-39]. In addition, contrast may also help reveal dilated venous structures that are masked by artifact or mass effect from cord swelling, are less visible due to small shunt volumes, or are otherwise too small to be found without contrast [3, 24, 39]. Furthermore, gadolinium is useful in the imaging evaluation and diagnosis of suspected tumors, and thus may further aid in differentiating SDAVF from other mimics [39]. The dilated serpentine veins may also be more adequately revealed on myelographic or heavily weighted $\mathrm{T} 2$ sequences, including CISS, FIESTA, and 3D-TSE, than on standard T2 weighted imaging (T2WI), as the flow voids are frequently obscured by mass effect or pulsation artifact [20, 24]. Both myelographic and heavily T2 weighted sequences are useful because they are volumetric, have good spatial resolution, and are not dependent on flow [24].

Due to the relatively nonspecific nature of these imaging findings on MRI, SDAVF remains difficult to diagnose. The most common finding of the central T2 hyperintensity is also often seen on MRI in a variety of other spinal pathologies including infection, demyelination, inflammation, and tumor. While the imaging finding most specific for SDAVF is enlarged pial veins, this feature is observed less frequently [36]. Gilbertson et al reported finding central T2 hyperintensity in $100 \%$ of patients with SDAVF, but flow voids in only $35 \%$ of patients on $\mathrm{T} 1$ weighted imaging, and $45 \%$ on T2WI [37]. However, a combination of these subtle findings may suggest SDAVF. Lindenholz et al considered the hallmark MRI findings to include T2 hyperintensity, conus hyperintensity, flow voids, and patchy cord enhancement, and they found $88 \%$ of patients in their study to have all four of these findings [38].

If MRI findings are suggestive of SDAVF, obtaining an MRA is advised as it is often able to accurately predict the spinal level of the SDAVF within a narrow range [12, 24, $34,38]$. In one study, use of MRA was able to reveal the exact level of the SDAVF in $42.9 \%$ of patients (18 out of 44 ), and within 2 levels in $59.5 \%$ of patients ( 25 out of 44 ) [12]. This localization of the fistula allows subsequent DSA to be more targeted, reducing the amount of time, radiation, and contrast needed [24, 34]. Although MRI and MRA findings may point towards SDAVF, DSA is considered to be the gold standard for diagnosing SDAVF, and is more sensitive than MRA [3, 11, 15]. Important angiographic findings include early filling of radicular veins and delayed venous return. Angiography is sometimes the only imaging modality that can reveal the SDAVF, as some patients have negative findings on MR studies [39].

\section{Differentiating SDAVF from other similar clinical presentations}

There are numerous entities that mimic SDAVF on imaging studies. The spinal cord edema present in SDAVF that is visualized as central hyperintensity on T2WI may also be seen in spinal cord tumor, myelitis, anterior spinal artery infarction, and persistent central canal [15]. However, in SDAVF, the hyperintensity is typically homogenous, extends over multiple spinal levels, frequently involves the conus, and is surrounded by a rim of hypointensity $[15,36]$. In addition, the flow voids found on T2WI representing dilated serpentine perimedullary veins may also appear similar to the flow voids found in spinal stenosis, as this condition can also cause venous dilation. However, in the case of spinal stenosis, the finding of centromedullary $\mathrm{T} 2$ hyperintensity should be absent.

There have been multiple reports of patients with SDAVF initially misdiagnosed as tumor, as MRI features of both SDAVF and tumor include spinal cord enlargement, T2 hyperintensity, and contrast enhancement [16, 40, 41]. This often results in the patient undergoing biopsy of the suspected lesion. However, certain pathological features characteristic of venous congestive myelopathy may distinguish SDAVF from tumor. These features include a large amount of small hyalinized vessels, perivascular hemosiderin deposits, vascular thrombosis, necrosis, and Rosenthal fibers $[16,41]$. In a study of patients suspected to have SDAVF by Rodriguez et al, the presence of thrombosis was the major distinguishing pathological feature between patients found to have an SDAVF and those ultimately found to have no SDAVF [16].

As SDAVF is frequently misdiagnosed as other disorders, including polyneuropathies or polyradiculopathies, it is also important to keep in mind the clinical features that distinguish it from these disorders. Distinct clinical features of SDAVF include the ascending nature of symptoms, with sensory loss moving all the way up to involve the perianal area. By comparison, polyneuropathies usually involve the distal extremities, including the hands. Bowel and bladder dysfunction, seen in SDAVF, do not occur in polyneuropathies or radiculopathies [13, 14]. In addition, symptoms are typically asymmetric in SDAVF, while neuropathy symptoms are more likely to be symmetrical. Furthermore, if the spinal cord above the conus becomes involved, upper motor neuron signs will occur, making it clear that the pathology is not peripheral in nature [14].

\section{Conclusion}

In the case of our patient, she first presented to our hospital with moderate bilateral lower extremity weakness, 
dysesthesias, numbness and tingling. Her MRI showed findings suggestive of SDAVF in the thoracolumbar region, including cord edema within the conus and serpiginous flow voids. Although she underwent an attempted angiogram that ultimately could not be completed, clinical suspicion for SDAVF remained high, based on these MRI findings. However, the patient declined to undergo further testing or intervention, opting to be discharged to a rehabilitation center, despite being informed that she would most likely continue to deteriorate neurologically. Unfortunately, approximately 1 month later, she returned to our hospital, completely paraplegic. MRI at this time showed extensive signal abnormality, with heterogeneous enhancement of the thoracolumbar spine. In the context of known SDAVF, these findings were suggestive of cord infarction. Over a course of 1 month, we were able to observe the progressive clinical and radiological deterioration of SDAVF. In addition, in reviewing the patient's images from years prior, we could retrospectively observe this SDAVF as it began to develop.

Thus, through this series of images, we demonstrate the radiographic progression of SDAVF from early in the course of the disease until its ultimate progression to venous congestive myelopathy and spinal cord infarction. In addition, we demonstrate the importance of keeping SDAVF within the differential of clinical symptoms and imaging findings such as these. The late stage imaging findings of SDAVF suggestive of infarction could also represent other disease processes and often leads to misdiagnosis as neoplastic or inflammatory processes. Therefore, given the fact that patients may present to a physician at any point along the time course of their disease progression, SDAVF must be considered as a possible etiology in the context of similar symptoms and MRI findings. This is especially important because SDAVF represents a potentially treatable cause of myelopathy, and recognizing it early may protect the patient from multiple unnecessary tests, disease progression, and ultimately severe neurologic disability.

Acknowledgements This research did not receive any specific grant from funding agencies in the public, commercial, or not-for-profit sectors.

\section{Compliance with ethical standards}

Conflict of interest The authors declare that they have no conflict of interest.

\section{References}

1. Bao YH, Ling F. Classification and therapeutic modalities of spinal vascular malformations in 80 patients. Neurosurgery. 1997;40:75-81.
2. Endo T, Endo H, Sato K, Matsumoto Y, Tominaga T. Surgical and endovascular treatment for spinal arteriovenous malformations. Neurol Med Chir. 2016;56:457-64.

3. Krings T, Lasjaunias PL, Hans FJ, Mull M, Nijenhuis RJ, Alvarez $\mathrm{H}$, et al. Imaging in spinal vascular disease. Neuroimaging Clin $\mathrm{N}$ Am. 2007;17:57-72.

4. Anson JA, Spetzler RF. Classification of spinal arteriovenous malformations and implications for treatment. BNI Q. 1992;8:2-8.

5. Ropper Ae, Gross BA, Du R. Surgical treatment of Type I spinal dural arteriovenous fistulas. Neurosurg Focus. 2012;32:E3.

6. Spetzler RF, Detwiler PW, Riina HA, Porter RW. Modified classification of spinal cord vascular lesions. J Neurosurg. 2002;96:145-56.

7. Medel R, Crowley RW, Dumont AS. Endovascular management of spinal vascular malformations: history and literature review. Neurosurg Focus. 2009;26:E7.

8. Ferch RD, Morgan MK, Sears WR. Spinal arteriovenous malformations: a review with case illustrations. J Clin Neurosci. 2001;8:299-304.

9. Oldfield EH. Introduction: spinal vascular malformations. Neurosurg Focus. 2009;26:E1.

10. Gemmete JJ, Chaudhary N, Elias AE, Toma AK, Pandey AS, Parker RA, et al. Spinal dural arteriovenous fistulas: clinical experience with endovascular treatment as a primary therapy at 2 academic referral centers. Am J Neuroradiol. 2013;34:1974-9.

11. Jellema K, Tijssen CC, van Gijn J. Spinal dural arteriovenous fistulas: a congestive myelopathy that initially mimics a peripheral nerve disorder. Brain. 2006;129:3150-64.

12. Muralidharan R, Saladino A, Lanzino G, Atkinson JL, Rabinstein AA. The clinical and radiological presentation of spinal dural arteriovenous fistula. Spine. 2011;36:E1641-47.

13. Van Dijk JM, TerBrugge KG, Willinsky RA, Farb RI, Wallace MC. Multidisciplinary management of spinal dural arteriovenous fistulas: clinical presentation and long-term follow-up in 49 patients. Stroke. 2002;33:1578-83.

14. Jellema K. Spinal dural arteriovenous fistulas: clinical features in 80 patients. J Neurol Neurosurg Psychiatr. 2003;74 (10): 1438-440.

15. Jeng Y, Chen DY, Hsu HL, Huang YL, Chen CJ, Tseng YC. Spinal dural arteriovenous fistula: imaging features and its mimics. Korean J Radiol. 2015;16(5):1119

16. Rodriguez FJ, Crum BA, Krauss WE, Scheithauer BW, Giannini C. Venous congestive myelopathy: a mimic of neoplasia. Mod Pathol Mod Pathol. 2004;18(5):710-18.

17. Oldfield EH. Surgical treatment of spinal dural arteriovenous fistulas. Semin Cerebrovasc Dis Stroke. 2002;2:209-26.

18. Jahan R, Vinuela F. Vascular anatomy, pathophysiology and classification of spinal vascular malformations of the spinal cord. Semin Cerebrovasc Dis Stroke. 2002;2:186-200.

19. Dehdashti AR, Da Costa LB, terBrugge KG, Willinsky RA, Tymianski M, Wallace MC. Overview of the current role of endovascular and surgical treatment in spinal dural arteriovenous fistulas. Neurosurg Focus. 2009;26:E8.

20. Krings T, Geibprasert S. Spinal dural arteriovenous fistulas. AJNR AM J Neuroradiol. 2009;30:639-48.

21. Patsalides A, Santillan A, Knopman J, Tsiouris AJ, Riina HA, Gobin YP. Endovascular management of spinal dural arteriovenous fistulas. J NeuroIntervent Surg. 2011;3:80-84.

22. Kataoka H, Miyamoto S, Nagata I, Ueba T, Hashimoto N. Venous congestion is a major cause of neurological deterioration in spinal arteriovenous malformations. Neurosurgery. 2001;48:1224-30.

23. Da Costa L, Dehdashti AR, Terbrugge KG. Spinal cord vascular shunts: spinal cord vascular malformations and dural arteriovenous fistulas. Neurosurg Focus. 2009;26:E6.

24. Morris JM. Imaging of dural arteriovenous fistula. Radiol Clin North Am. 2012;50(4):823-39. 
25. Saladino A, Atkinson JL, Rabinstein AA, Piepgras DG, Marsh WR, Krauss WE, et al. Surgical treatment of spinal dural arteriovenous fistulae: a consecutive series of 154 patients. Neurosurgery. 2010;67:1350-7.

26. Krings T, Thron AK, Geibprasert S, Agid R, Hans FJ, Lasjaunias PL, et al. Endovascular management of spinal vascular malformations. Neurosurg Rev. 2010;33:1-9.

27. Narvid J, Hetts SW, Larsen D, Neuhaus J, Singh TF, McSwain H, et al. Spinal dural arteriovenous fistulae: clinical features and long-term results. Neurosurgery. 2008;62:159-66 (discussion166-67).

28. Aminoff MJ, Logue V. Clinical features of spinal vascular malformations. Brain. 1974;97:197-210.

29. Foix $\mathrm{CH}$, Alajouanine T. La myelite necrotique subaigue. Rev Neurol. 1926;46:1-42.

30. Schrader V, Koenig E, Thron A, Dichgans J. Neurophysiological characteristics of spinal arteriovenous malformations. Electromyogr Clin Neurophysiol. 1989;29:169-77.

31. Steinmetz MP, Chow MM, Krishnaney AA, Andrews-Hinders D, Benzel EC, Masaryk TJ, et al. Outcome after the treatment of spinal dural arteriovenous fistulae: a contemporary singleinstitution series and meta-analysis. Neurosurgery. 2004;55:77-88.

32. Cenzato M, Debernardi A, Stefini R, D'Aliberti G, Piparo M, Talamonti G, et al. Spinal dural arteriovenous fistulas: outcome and prognostic factors. Neurosurg Focus. 2012;32:E11.

33. Sivakumar W, Zada G, Yashar P, Giannotta SL, Teitelbaum G, Larsen DW. Endovascular management of spinal dural arteriovenous fistulas. A review. Neurosurg Focus. 2009;26:E15.
34. Saraf-Lavi E, Bowen BC, Quencer RM, Sklar EML, Holz A, Falcone S, et al. Detection of spinal dural arteriovenous fistulae with $\mathrm{mr}$ imaging and contrast-enhanced $\mathrm{mr}$ angiography: sensitivity, specificity, and prediction of vertebral level. Am J Neuroradiol. 2002;23:858-67.

35. Luetmer PH, Lane JI, Gilbertson JR, Bernstein MA, Huston J,3rd, Atkinson JL. Preangiographic evaluation of spinal dural arteriovenous fistulas with elliptic centric contrast-enhanced $\mathrm{mr}$ angiography and effect on radiation dose and volume of iodinated contrast material. Am J Neuroradiol. 2005;26(4):711-18.

36. Hurst RW, Grossman RI. Peripheral spinal cord hypointensity on t2-weighted $\mathrm{mr}$ images: a reliable imaging sign of venous hypertensive myelopathy. Am J Neuroradiol. 2000;4(21):781-86.

37. Gilbertson RJ, Miller GM, Goldman MS, Marsh WR. Spinal dural arteriovenous fistulas: MR and myelographic findings. Spinal Dural Arter Fistula. 1995;16:2049-57.

38. Lindenholz A, Terbrugge KG, van Dijk JM, Farb RI. The accuracy and utility of contrast-enhanced MR angiography for localization of spinal dural arteriovenous fistulas: the toronto experience. Eur Radiol Eur Radiol. 2014;24(11):2885-894.

39. Dillon WP, Norman D, Newton TH, Bolla K, Mark A. Intradural spinal cord lesions: Gd-DTPA-enhanced MR imaging. Radiology. 1989;170(1):229-37.

40. Roccatagliata L, Centanaro F, Castellan L. Venous congestive myelopathy in spinal dural arteriovenous fistula mimicking neoplasia. Neurol Sci Neurol Sci. 2007;28(4):212-15.

41. Hurst RW, Kenyon LC, Lavi E, Raps EC, Marcotte P. Spinal dural arteriovenous fistula: the pathology of venous hypertensive myelopathy. Neurology. 1995;45(7):1309-313. 